\title{
Spontaneous stage differentiation of mouse-virulent Toxoplasma gondii RH parasites in skeletal muscle cells: an ultrastructural evaluation
}

\author{
Marialice da Fonseca Ferreira-da-Silva ${ }^{1,3}$, Renata Mendonça Rodrigues ${ }^{1}$, \\ Elisabete Ferreira de Andrade', Laís de Carvalho², Uwe Gross ${ }^{3}$, Carsten G K Lüder³, \\ Helene Santos Barbosa ${ }^{1 /+}$
}

\begin{abstract}
${ }^{1}$ Laboratório de Biologia Estrutural, Instituto Oswaldo Cruz-Fiocruz, Av. Brasil 4365, 21040-361 Rio de Janeiro, RJ, Brasil ${ }^{2}$ Laboratório de Cultura de Células, Departamento de Histologia e Embriologia, Universidade do Estado do Rio de Janeiro, Rio de Janeiro, RJ, Brasil ${ }^{3}$ Institute for Medical Microbiology, Georg-August University, Kreuzbergring 57, 37075, Göttingen, Germany
\end{abstract}

Although the predilection for Toxoplasma gondii to form cysts in the nervous system and skeletal and heart muscles has been described for more than fifty years, skeletal muscle cells (SkMCs) have not been explored as a host cell type to study the Toxoplasma-host cell interaction and investigate the intracellular development of the parasite. Morphological aspects of the initial events in the Toxoplasma-SkMC interaction were analysed and suggest that there are different processes of protozoan adhesion and invasion and of the subsequent fate of the parasite inside the parasitophorous vacuole $(P V)$. Using scanning electron microscopy, Toxoplasma tachyzoites from the mouse-virulent RH strain were found to be attached to SkMCs by the anterior or posterior region of the body, with or without expansion of the SkMC membrane. This suggests that different types of parasite internalization occurred. Asynchronous multiplication and differentiation of $\mathrm{T}$. gondii were observed. Importantly, intracellular parasites were seen to display high amounts of amylopectin granules in their cytoplasm, indicating that tachyzoites of the RH strain were able to differentiate spontaneously into bradyzoites in SkMCs. This stage conversion occurred in approximately 3\% of the PVs. This is particularly intriguing as tachyzoites of virulent Toxoplasma strains are not thought to be prone to cyst formation. We discuss whether biological differences in host cells are crucial to Toxoplasma stage conversion and suggest that important questions concerning the host cell type and its relevance in Toxoplasma differentiation are still unanswered.

Key words: Toxoplasma gondii - stage conversion - T. gondii-host cell interaction - skeletal muscle cell - tachyzoite - bradyzoite

Toxoplasma gondii is an obligate intracellular parasite that exists in two stages in the host: tachyzoites and bradyzoites. The tachyzoites replicate rapidly, are present in the acute infection and can infect any eukaryotic cell. The bradyzoites replicate slowly and forms tissue cysts primarily in the brain and in muscle cells during latent infections (Dubey et al. 1998).

Even 100 years after Toxoplasma was initially described, there is still some disagreement regarding whether the type of host cell is critical for cyst formation. Some studies suggest that the choice of cell type does not appear to have a large effect on the tachyzoitebradyzoite conversion or on cyst-forming ability (Lindsay et al. 1991), while others propose that some host cell types may provide the optimal metabolic environment for cyst development (Jones et al. 1986, Radke et al. 2006, Ferreira-da-Silva et al. 2008a).

Financial support: CNPq, PAPES IV (Fiocruz), Fundação Carlos Chagas Filho - FAPERJ, Instituto Oswaldo Cruz, Fiocruz, Bundesministerium für Bildung und Forschung (TOXONET01, Grant 01 K1 0766 to CGK Lüder), Deutscher Akademischer Austauschdienst (to MF Ferreira-da-Silva), CAPES (to RM Rodrigues)

+ Corresponding author: helene@ioc.fiocruz.br

Received 10 October 2008

Accepted 9 March 2009
Little information exists on the host cell type of the cyst stage in humans. The understanding of the intracellular fate of $T$. gondii, especially during the tachyzoitebradyzoite conversion, and the cystogenesis process is limited to the context of in vitro studies. Unfortunately, the cellular models employed in the majority of stage conversion investigations are not cells involved in the development of toxoplasmosis in vivo. The easy handling and maintenance of the cells and their availability were and still are the main concerns that led scientists to invest in in vitro cell lines (McHugh et al. 1994).

Our group has used primary cultures of skeletal muscle cells (SkMCs) as a cellular model for experimental toxoplasmosis studies of (i) the molecular events of the parasite-host cell interaction, (ii) the formation of the parasitophorous vacuole (PV) and (iii) the intracellular fate of parasites (Monteiro-Leal et al. 1998, Andrade et al. 1999, 2001, Barbosa et al. 1999, 2005, Ferreira-daSilva et al. 2008a, Guimarães et al. 2008). This proposal aims to explore the use of SkMCs for the analysis of the early and late events of Toxoplasma interaction with its host cell.

\section{MATERIALS AND METHODS}

The procedures were performed in accordance with the guidelines established by the Colégio Brasileiro de Experimentação Animal, by the Fundação Oswaldo Cruz-Fiocruz Committee of Ethical for the Use of Animals (license CEUA 0229-04) and by the Guidelines on 
the Care and Use of Animals for Experimental Purposes and Infectious Agents.

Culture of primary SkMCs - SkMCs were obtained from the thigh muscles of 18-day-old mouse embryos. The tissues were minced and incubated for $7 \mathrm{~min}$ with $0.05 \%$ trypsin and $0.01 \%$ versene diluted in phosphatebuffered saline (PBS), pH 7.2. After 5-8 dissociation steps, enzyme digestion was stopped by adding $10 \%$ foetal bovine serum. The cellular suspension was centrifuged for $7 \mathrm{~min}$ at $650 \mathrm{~g}$ and resuspended in Dulbecco's modified Eagle medium (DMEM) supplemented with $10 \%$ horse serum, $5 \%$ foetal calf serum, $2 \%$ chick embryo extract, $1 \mathrm{mM} \mathrm{L}$-glutamine, $1,000 \mathrm{U} / \mathrm{mL}$ penicillin and $50 \mu \mathrm{g} / \mathrm{mL}$ streptomycin. The cells then were incubated for $30 \mathrm{~min}$ at $37^{\circ} \mathrm{C}$ in a $5 \% \mathrm{CO} 2$ atmosphere. After incubation, the culture flask was gently shaken to release the non-attached cells and the supernatant enriched with myoblasts was seeded in $0.02 \%$ gelatine-treated $35 \mathrm{~mm}$ culture plates (for electron microscopy studies). The cultures were incubated at $37^{\circ} \mathrm{C}$ in a $5 \% \mathrm{CO} 2$ atmosphere and the medium was replaced every two days. The culture was maintained for three-five days to obtain muscle fibres (Barbosa et al. 2000).

Parasite isolation - Tachyzoites from the mouse-virulent type I RH strain of $T$. gondii were maintained by intraperitoneal passaging in female Swiss mice. The parasites were collected $72 \mathrm{~h}$ after infection in PBS, $\mathrm{pH}$ 7.2. The suspension obtained from infected mice was centrifuged at $200 \mathrm{~g}$ for $10 \mathrm{~min}$ at $\mathrm{rt}$ to completely remove cells and debris. The supernatant, which contained only the parasites, was collected and centrifuged at $1,000 \mathrm{~g}$ for 10 min. The resulting pellet was washed two or three times with PBS, pH 7.2, resuspended in DMEM and used in the interaction assays. SkMCs were infected with $T$. gondii tachyzoites at a parasite to host cell ratio of 5:1.

Scanning electron microscopy (SEM) - SkMCs were infected with a $T$. gondii tachyzoites RH strain (5:1 parasite-host cell ratio) for periods of $15 \mathrm{~min}-4 \mathrm{~h}$ at $37^{\circ} \mathrm{C}$ in a $5 \% \mathrm{CO}_{2}$ atmosphere. After this period, cells were fixed for $30 \mathrm{~min}$ at $\mathrm{rt}$ in $2.5 \%$ glutaraldehyde (GA) diluted in $0.1 \mathrm{M}$ Na-cacodylate buffer. After washing in the same buffer, the cells were post-fixed for $30 \mathrm{~min}$ at $\mathrm{rt}$ in $1 \%$ osmium tetroxide $\left(\mathrm{OsO}_{4}\right)$. The resulting material, after washing in buffer, was then dehydrated using an ascending acetone series, dried by the critical point method with $\mathrm{CO}_{2}$, mounted with silver cellotape on aluminium stubs and coated with a $20 \mathrm{~nm}$-thick layer of gold. The samples were examined with a Zeiss DSM 940 microscope.

Transmission electron microscopy (TEM) - SkMCs were infected with $T$. gondii tachyzoites (5:1 parasitehost cell ratio) and incubated at $37^{\circ} \mathrm{C}$ in a $5 \% \mathrm{CO}_{2}$ atmosphere. After different periods of infection $(1,18$ and 48 h), the cell cultures were washed three times for $10 \mathrm{~min}$ with PBS and fixed for $1 \mathrm{~h}$ at $4{ }^{\circ} \mathrm{C}$ in $2.5 \%$ GA diluted in $0.1 \mathrm{M}$ Na-cacodylate buffer containing $3.5 \%$ sucrose and $2.5 \mathrm{mM} \mathrm{CaCl}_{2}, \mathrm{pH}$ 7.2. After fixation, the cells were washed in the same buffer and then post-fixed for $30 \mathrm{~min}$ at $\mathrm{rt}$ in $1 \% \mathrm{OsO}_{4}$ diluted in $0.1 \mathrm{M} \mathrm{Na}$-cacodylate buffer. The cells were then washed in the same buffer, scraped at $4^{\circ} \mathrm{C}$ from the plastic dish and centrifuged for $5 \mathrm{~min}$ at $10,000 \mathrm{~g}$. Next, the cells were dehydrated in graded acetone and embedded in Epoxy resin. Thin sections were stained with uranyl acetate and lead citrate and examined under a Zeiss EM10C TEM. The percentage of bradyzoite-containing PVs was determined by counting at least 100 randomly selected PVs in thin sections.

\section{RESULTS}

Ultrastructural analysis by SEM showed highly differentiated SkMCs that displayed a typical fusiform shape (Fig. 1). Early in the myotube host-pathogen interaction, Toxoplasma tachyzoites were seen in close contact with the SkMCs, sometimes by the anterior (apical portion) or posterior region of their bodies (Figs 1, 2A). The images obtained during the interaction between $T$. gondii and SkMCs indicated three different invasion mechanisms: (i) active penetration, where the apical part of the parasite (specifically the conoid region) clearly depicted a torsion of the parasite's body, as visualized by the subpellicular microtubule arrangement (Figs 1, 2A), (ii) phagocytosis, where the muscle cell engulfs the parasite (Fig. 2A) and (iii) expansion of the host cell membrane as a tunnel-like structure, as previously observed by MacLaren et al. (2004) (Fig. 2B). Examination of ultrathin sections confirmed these two last events, showing the engulfment of a T. gondii tachyzoite (Fig. 3).

Analysis of later events of tachyzoite development in SkMCs revealed developing intracellular parasites within $\mathrm{PV}$ and muscle cells that displayed the typical striation characteristics of myofibres (Fig. 4). Ultrastructural studies of SkMC cultures infected with tachyzoites revealed typical bradyzoite forms within PVs, as identified by their large amount of amylopectin granules in the cytoplasm, after $18 \mathrm{~h}$ (Figs 5, 6). Each single parasite was observed to be isolated in one vacuole, without apparent signs of cellular division (Figs 5, 6). Some cells presented two PVs, one containing bradyzoite forms and the other tachyzoites, demonstrating that a non-synchronized process occurs during this conversion (Figs 6, 7). Fig. 7 shows another aspect of this asynchrony after $24 \mathrm{~h}$ of interaction, as illustrated by three vacuoles that display a bradyzoite form, four tachyzoites and one tachyzoite, respectively.

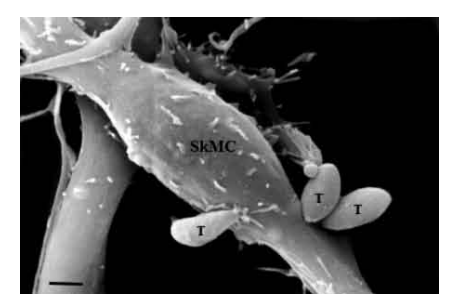

Fig. 1: scanning electron microscopy showing the interaction of Toxoplasma gondii tachyzoites with skeletal muscle cells after $30 \mathrm{~min}$ of interaction. Highly differentiated muscle cell displaying the typical fusiform shape. Tachyzoites (T) are seen adhered to the cell surface by the apical portion. Note that the tachyzoite at the right side of the micrograph $\left(^{*}\right)$ shows clearly torsion of its body, observing its subpellicular microtubules disposed in counter-clockwise direction. Interaction between host cell filopodium and parasite at the left side are seen. $\operatorname{Bar}=1 \mu \mathrm{m}$. 


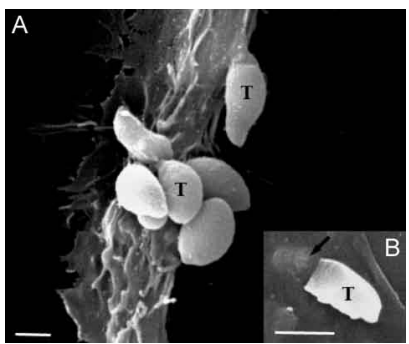

Fig. 2A: tachyzoites (T) of Toxoplasma gondii and skeletal muscle cell by scanning electron microscopy. Several parasites are attached to the host cell surface; B: note that the membrane of the host cell partially covers the parasite (arrow) after 30 min of interaction, suggesting that the tachyzoite is being phagocyted by the skeletal muscle cell. Bar $=$ $0.5 \mu \mathrm{m}$.

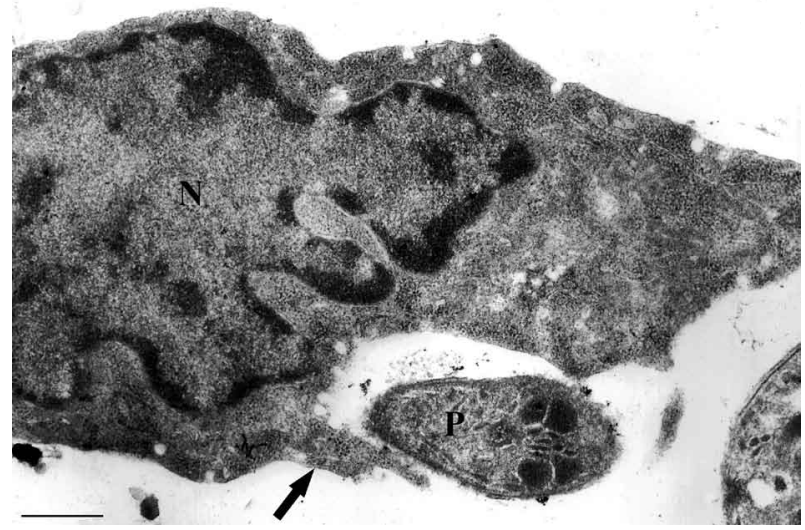

Fig. 3: tachyzoites of Toxoplasma gondii and skeletal muscle cell seen by transmission electron microscopy. SkMC emitting pseudopode (arrow) toward the parasite (P) after $1 \mathrm{~h}$ of interaction. N: nuclei. Bar $=0.5 \mu \mathrm{m}$.

Analysis of infected cells after $48 \mathrm{~h}$ of interaction revealed several parasites within the $\mathrm{PV}$, in a classic process of cellular division by endodyogeny (Fig. 8). Transitory forms were observed in this PV, where some parasites had an accumulation of amylopectin granules at the posterior region of the body, indicative of tachyzoite-bradyzoite conversion. Quantitative ultrastructural analysis showed that the conversion of the $T$. gondii tachyzoites into bradyzoites occurred in 3\% of the PVs.

\section{DISCUSSION}

In the present study, we investigated the interaction between Toxoplasma tachyzoites and SkMCs in vitro. SkMCs are a host cell type known to be an important target cell for this parasite and are where tissue cysts are commonly formed.

Toxoplasma does not have specialized organelles for motility, such as cilia or flagella, yet they are able to move along surfaces by gliding and actively invade host cells by a microfilament-based motility mechanism (Laliberté \& Carruthers 2008). High-resolution SEM has shown three distinct processes of $T$. gondii tachyzoite penetration into neutrophils, including formation of surface projections of the host cell, depression of the host

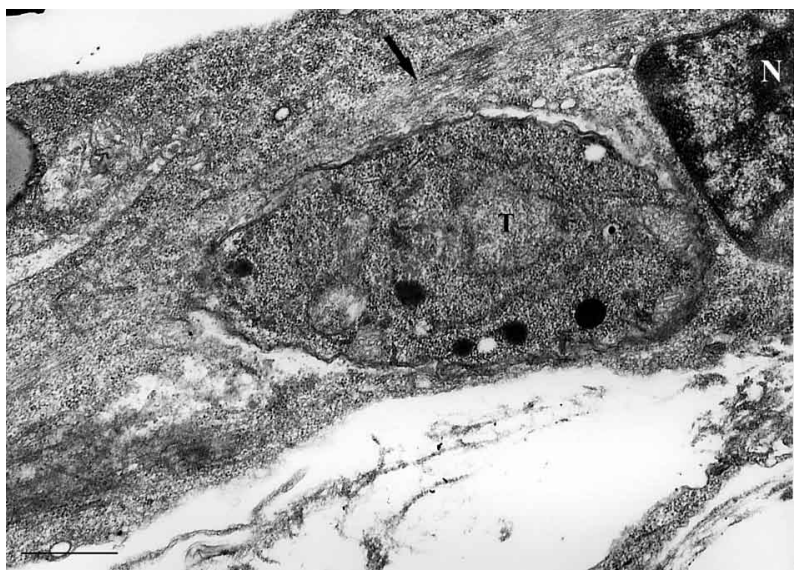

Fig. 4: ultrastructural image showing a parasitophorous vacuole containing one single tachyzoite of Toxoplasma in SkMC after $18 \mathrm{~h}$ of infection. Longitudinal thin section displaying the typical microfilaments organization in the muscle fibers (arrow). N: nuclei; T: tachyzoite.
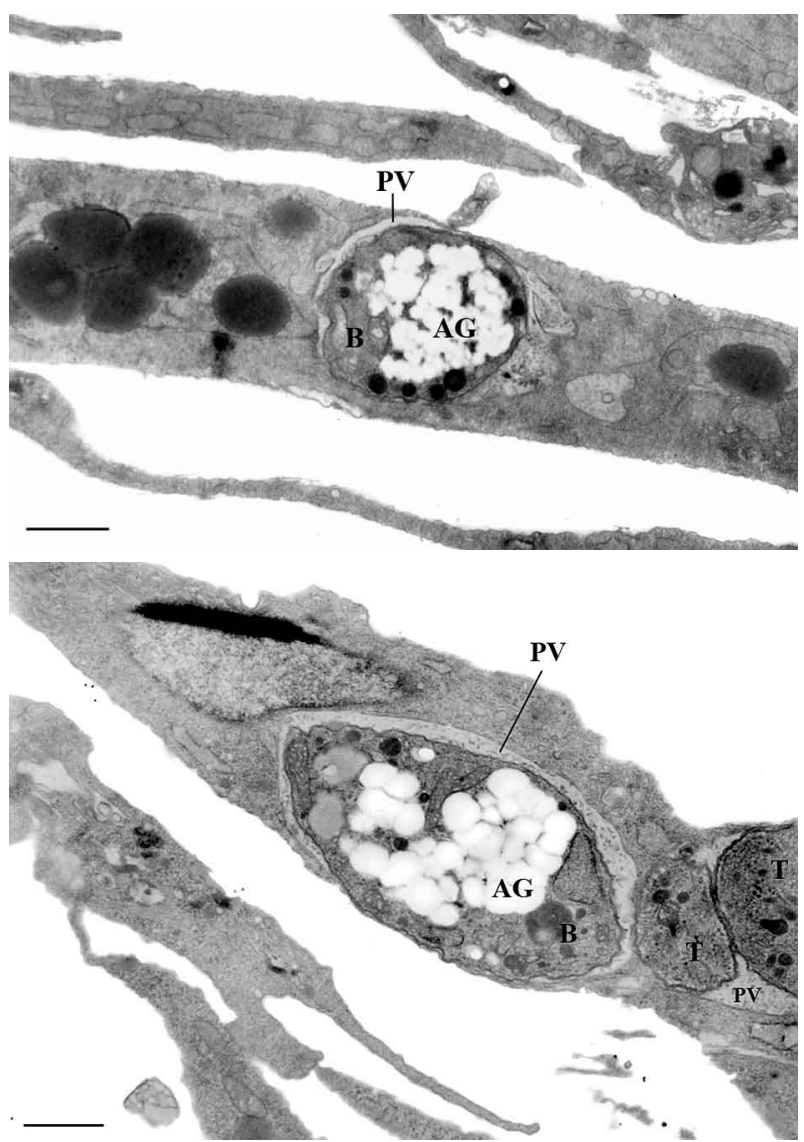

Figs 5, 6: ultrastructural aspects displaying SkMC after $18 \mathrm{~h}$ of infection with tachyzoites. Both figures show typical bradyzoites (B) identified by their great amount of amylopectin granules (AG) in their cytoplasm and, in the fig. 6 , another vacuole containing tachyzoites (T). PV: parasitophorous vacuole. $\mathrm{Bar}=0.5 \mu \mathrm{m}$.

cell surface and also a tunnel-like structure formed by a special projection of the host cell (MacLaren et al. 2004). Our analysis of the initial association of $T$. gondii with highly differentiated SkMC cultures revealed that these 


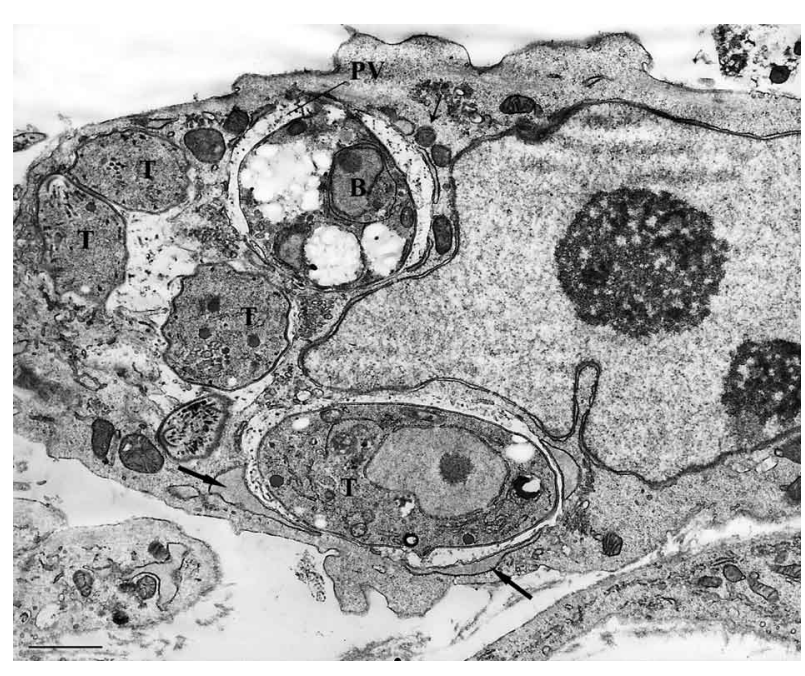

Fig. 7: ultrastructural analysis showing intracellular Toxoplasma gondii after $24 \mathrm{~h}$ of interaction with SkMC. The parasites are seen surrounded by a membrane of a typical parasitophorous vacuole (PV) containing a bradyzoite (B) and other vacuoles with tachyzoites (T). Profiles of the endoplasmatic reticulum (thin arrow) and mitochondria (large arrow) are associated with the membrane of the vacuole. Bar $=0.5 \mu \mathrm{m}$.

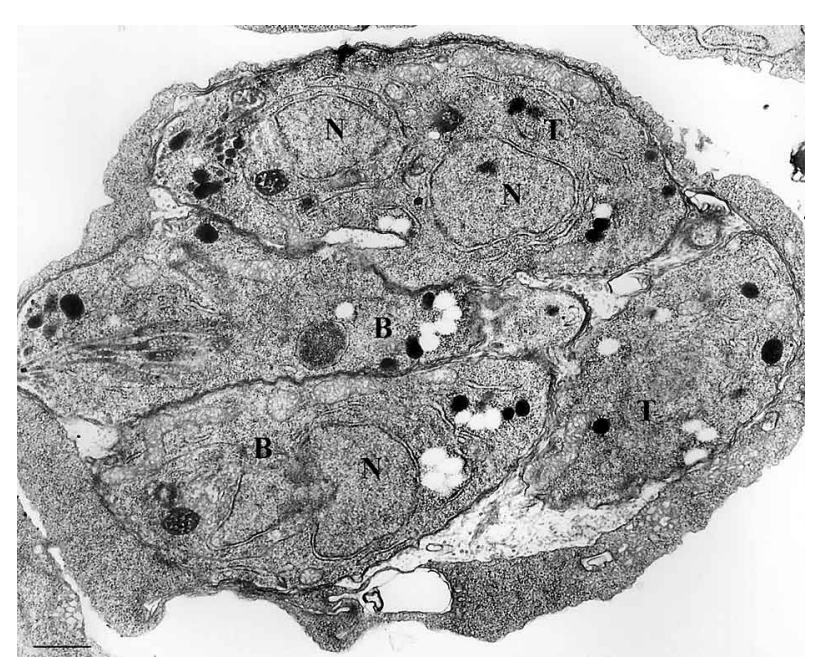

Fig. 8: transmission electron microscopy of SkMC after $48 \mathrm{~h}$ of infection with Toxoplasma gondii tachyzoites. Parasites (P) in process of cellular division (endodyogeny) can be observed. The PV contains bradyzoites (B) with some amylopectin granules together with tachyzoites $(\mathrm{T})$ confirming that transitory forms can be found in the same PV. Bar $=0.5 \mu \mathrm{m}$.

events also occurred during parasite attachment and the invasion process into SkMCs. The active invasion by $T$. gondii into host cells has been well established, but other mechanisms involving active participation of the host cell have also been suggested (reviewed in De Souza 2009). Further studies are required to resolve these other molecular mechanisms and to unravel their impact on the parasite-host interaction.

TEM examination of the interaction between SkMCs and a mouse-virulent (RH) T. gondii strain revealed a spontaneous conversion of the $T$. gondii from the tachyzoite stage to the bradyzoite stage. This is intriguing because mouse-virulent strains of $T$. gondii, including the RH strain, are not associated with cyst formation. Instead, these parasites rapidly multiplied and lysed host cells in previous experiments performed under standard conditions, i.e., at physiological temperature and $\mathrm{pH}$ and in the absence of drugs or any other hostile treatment (De Champs et al. 1997, Ferreira-da-Silva et al. 2008a). However, under stress conditions, i.e., at $\mathrm{pH} 8.0$, the $\mathrm{RH}$ strain was able to form cysts that were surrounded by a 0.5-1-micron irregular wall and contained bradyzoites (De Champs et al. 1997). In addition, RH cystogenesis also occurred in non-immunized Fischer rats and led to a concomitant attenuation of RH pathogenicity in nonimmunized mice (Lecomte et al. 1992). Importantly, we now provide ultrastructural evidence that $\mathrm{RH}$ parasites are also able to differentiate spontaneously into bradyzoites in SkMCs. These data are in agreement with the spontaneous conversion observed by our group after using tachyzoites of the mouse-avirulent NTE strain to infect primary SkMCs (Ferreira-da-Silva et al. 2008b) and a L6.C10 myoblast cell line (Ferreira-da-Silva et al. 2008a). In a comparative study using fibroblasts and SkMCs, we found evidence that the cell type may be critical for the relative level of $T$. gondii stage conversion (Ferreira-da-Silva et al. 2008b). It remains unclear why and under which conditions tachyzoites of some strains are able to differentiate in cell culture in the absence of any obvious inductive agents (Bohne et al. 1993, Soête et al. 1993, Lindsay et al. 1998). This is especially true for the RH strain that is considered less cystogenic than mouse-avirulent Toxoplasma strains. Understanding this process could help in designing new chemotherapeutic agents that act before the formation of tissue cysts.

One of the major questions in the field is whether spontaneous or induced stage conversion leads to or supports the intracellular development of the parasite (Dubey et al. 1998, Ferreira-da-Silva et al. 2008a, b). In addition, there is little information regarding the mechanisms of tissue cyst formation or how this correlates with the cell type in which the parasites develop and cause infection (Dubey et al. 1998). These issues are of particular importance as this may influence the maintenance of the tissue cyst in the host and may also provide knowledge concerning the route of parasite transmission via consumption of raw or undercooked meat containing Toxoplasma. Toxoplasma has been known to form cysts in the nervous system and skeletal and heart muscles for almost 50 years (Jacobs 1967). A key morphological difference between tachyzoites and bradyzoites, and their relative intracellular development, is the generation of a cyst wall. Bradyzoites are slowly multiplying organisms and often live inside their host cells for months or years. Bradyzoites accumulate amylopectin, a form of glycogen that serves as an energy source, in their cytoplasm (Gross et al. 1995, Sasono \& Smith 1998, Guimarães et al. 2003). This accumulation serves as an excellent morphological characteristic that can be used to study T. gondii conversion and cystogenesis in vitro. Our findings of tachyzoites and bradyzoites in the same host cell 
indicate that the conversion process is non-synchronized and confirm the results of a previous study in which macrophages were used as host cells (Bohne et al. 1993).

From the above remarks, a question remains unanswered: does the host cell type determine the intracellular fate of the parasite? The cause of tissue cyst formation in different organ systems and tissues is still unclear. Are neural and muscle cells more susceptible to this parasite than other cell types? Previous studies of $T$. gondii infection have used cell lines and tumour cells that are not usually present in the natural in vivo infection process during toxoplasmosis. Can the information on the cellular and molecular mechanisms of $T$. gondii invasion and its intracellular fate obtained in these studies be universally applied? Do physiological differences of host cells control the pathogenic mechanism(s) of $T$. gondii? Are there other factors, such as nutrient acquisition, local immunity or defence mechanisms, which influence the ability of the parasite to encyst?

\section{REFERENCES}

Andrade EF, Carvalho L, Barbosa, HS 1999. Association of lipids during Toxoplasma gondii-skeletal muscle cells interaction. Mem Inst Oswaldo Cruz 94 (Suppl. II): 59.

Andrade EF, Stumbo AC, Monteiro-Leal LH, Carvalho L, Barbosa HS 2001. Do microtubules around the Toxoplasma gondii-containing parasitophorous vacuole in skeletal muscle cells form a barrier for the phagolysosomal fusion? J Submicrosc Cytol Pathol 33: 337-341.

Barbosa HS, Andrade EF, Carvalho L 1999. Ultrastructural aspects of the Toxoplasma gondii-skeletal muscle cells interaction. Mol Biol Cell 10: 182.

Barbosa HS, Ferreira-da-Silva MFF, Guimarães EV, Carvalho L, Rodrigues RM 2005. Absence of vacuolar membrane involving Toxoplasma gondii during its intranuclear localization. J Parasitol 91: 182-184.

Barbosa HS, Pereira MCS, Meirelles MNL 2000. Protocolos de culturas primárias. In Doença de Chagas: manual para experimentação animal, T Araújo-Jorge, SL de Castro (orgs.), Editora FIOCRUZ, Rio de Janeiro, p. 297-313.

Bohne W, Heesemann J, Gross U 1993. Coexistence of heterogeneous populations of Toxoplasma gondii parasites within parasitophorous vacuoles of murine macrophages as revealed by a bradyzoite-specific monoclonal antibody. Parasitol Res 79: 485-487.

De Champs C, Imbert-Bernard C, Belmeguenai A, Ricard J, Pelloux H, Brambilla E, Ambroise-Thomas P 1997. Toxoplasma gondii: in vivo and in vitro cystogenesis of the virulent RH strain. $J$ Parasitol 83: 152-155.

De Souza W, DaMatta, Attias M 2009 Brazilian contribution for a better knowledge on the biology of Toxoplasma gondii. Mem Inst Oswaldo Cruz 104: 149-154.

Dubey JP, Lindsay DS, Speer CA 1998. Structures of Toxoplasma gondii tachyzoites, bradyzoites and sporozoites and biology and development of tissue cysts. Clin Microbiol Rev 11: 267-299.
Ferreira-da-Silva MF, Barbosa HS, Gross U, Lueder CGK 2008a. Stress-related and spontaneous differentiation of Toxoplasma gondii. Mol Biosystem 4: 824-834.

Ferreira-da-Silva MF, Takács AC, Barbosa HS, Gross U, Lueder CGK 2008b. Primary skeletal muscle cells trigger spontaneous Toxoplasma gondii tachyzoite-to-bradyzoite conversion at higher rates than fibroblasts. Int J Med Microbiol, in press.

Gross U, Bormuth H, Gaissmaier C, Dittrich C, Krenn V, Bohne W, Ferguson DJ 1995. Monoclonal rat antibodies directed against Toxoplasma gondii suitable for studying tachyzoite-bradyzoite interconversion in vivo. Clin Diagn Lab Immunol 2: 542-548.

Guimarães EV, Carvalho L, Barbosa HS 2003. An alternative technique to reveal polysaccharides in Toxoplasma gondii tissue cysts. Mem Inst Oswaldo Cruz 98: 915-917.

Guimarães EV, Carvalho L, Barbosa HS 2008. Primary culture of skeletal muscle cells as a model for studies of Toxoplasma gondii cystogenesis. J Parasitol 94: 72-83.

Jacobs L 1967. Toxoplasma and toxoplasmosis. Adv Parasitol 5: 1-45.

Jones TC, Bienz KA, Erb P 1986. In vitro cultivation of Toxoplasma gondii cysts in astrocytes in the presence of gamma interferon. Infect Immun 51: 147-156.

Laliberté J, Carruthers VB 2008. Host cell manipulation by the human pathogen Toxoplasma gondii. Cell Mol Life Sci 65: 1900-1915.

Lecomte V, Chumpitazi BF, Pasquier B, Ambroise-Thomas P, Santoro F 1992. Brain-tissue cysts in rats infected with the RH strain of Toxoplasma gondii. Parasitol Res 78: 267-269.

Lindsay DS, Dubey JP, Blagburn BL, Toivio-Kinnucan M 1991. Examination of tissue cyst formation by Toxoplasma gondii in cell cultures using bradyzoites, tachyzoites and sporozoites. J Parasitol 77: 126-132.

Lindsay DS, Toivio-Kinnucan MA, Blagburn BL 1998. Decoquinate induces tissue cyst formation by the RH strain of Toxoplasma gondii. Vet Parasitol 15: 75-81.

MacLaren A, Attias M, de Souza W 2004. Aspects of the early moments of interaction between tachyzoites of Toxoplasma gondii with neutrophils. Vet Parasitol 10: 301-312.

McHugh TD, Holliman RE, Butcher PD 1994. The in vitro model of tissue cyst formation in Toxoplasma gondii. Parasitol Today 7: 281-285.

Monteiro-Leal LH, Stumbo AC, Campanati L, Barbosa HS, Carvalho L 1998. Video-microscopy analysis of the interaction of Toxoplasma gondii with host cells. Mem Inst Oswaldo Cruz 93 (Suppl II): 113.

Radke JR, Donald RG, Eibs A, Jerome ME, Behnke MS, Liberator P, White MW 2006. Changes in the expression of human cell division autoantigen-1 influence Toxoplasma gondii growth and development. PLoS Pathog 10: e105.

Sasono PM and Smith JE 1998. Toxoplasma gondii: an ultrastructure of host cell invasion by the bradyzoite stage. Parasitol Res 84: 640-645.

Soête M, Fortier B, Camus D, Dubremetz JF 1993. Toxoplasma gondii: kinetics of bradyzoite-tachyzoite interconversion in vitro. Exp Parasitol 76: 259-264. 